\title{
Plasma B-Type Natriuretic Peptide Is Useful for Cardiovascular Risk Assessment in Community-Based Diabetes Subjects
}

\section{Comparison With Albuminuria}

\author{
Masayuki Onodera, ${ }^{1}$ MD, Motoyuki NaKamura, ${ }^{1}$ MD, Fumitaka Tanaka, ${ }^{1}$ MD, \\ Tomohiro TaKahashi, ${ }^{1}$ MD, Shinji MaKita, ${ }^{1}$ MD, Takenori Ishisone, ${ }^{1} \mathrm{MD}$, Yasuhiro Ishibashi, ${ }^{1} \mathrm{MD}$, \\ Kazuyoshi ITAI, ${ }^{2} \mathrm{PhD}$, Toshiyuki OnODA, ${ }^{2} \mathrm{MD}$, Masaki Ohsawa, ${ }^{2} \mathrm{MD}$, Kozo Tanno, ${ }^{2} \mathrm{MD}$, \\ Kiyomi Sakata, ${ }^{2}$ MD, Shinichi Omama, ${ }^{3}$ MD, Kuniaki Ogasawara, ${ }^{3}$ MD, Akira Ogawa, ${ }^{3}$ MD, \\ Toru Kuribayashi, ${ }^{4} \mathrm{PhD}$, Kentaro SaKamaki, ${ }^{6} \mathrm{MPH}$, and Akira OKayama, ${ }^{5} \mathrm{MD}$
}

\section{SUMMARY}

Diabetes mellitus (DM) is a strong risk factor for cardiovascular (CV) disease. Plasma B-type natriuretic peptide (BNP) levels are elevated in various types of cardiac diseases. Increased plasma BNP levels have been reported to be associated with CV risk in apparently healthy individuals. However, no studies have yet examined the specific value of plasma BNP for predicting CV incidence in unselected DM subjects in a community-based population.

In a community-based DM cohort $(n=1,059$, mean $=66$ years $)$, baseline BNP levels were determined, and CV events were followed and captured prospectively. The cohort was divided by plasma BNP quintiles. The Cox proportional hazard model was used to determine the relative hazard ratios (HR) among the quintiles. In addition, the effects of adding the plasma BNP or urine albumin-to-creatinine ratio (UACR) to an established CV risk scoring model was examined by calculating the area under the receiver operating characteristic (ROC) curve (AUC).

During the 5.7 year follow-up period, $\mathrm{CV}$ events were identified in 65 of the DM cohort. There was a significant association between plasma BNP levels and $\mathrm{CV}$ event rate $(P<0.001)$. HR was significantly increased in the highest quintile compared to the lowest (HR $=4.38$; 95\%CI 1.69 -11.84). The AUC generated from ROC analysis of the Framingham risk score for predicting general $\mathrm{CV}$ events was improved by adding BNP testing (from 0.66 to $0.74 ; P=0.05$ ), but not by adding UACR (from 0.66 to $0.67 ; P=0.49$ ).

In a community sample of people with DM, plasma BNP levels above the 80 percentile are directly associated with $\mathrm{CV}$ risk, and measurement of plasma BNP alone or in conjunction with an established CV risk score is of value in predicting CV events in these subjects. (Int Heart J 2012; 53: 176-181)

Key words: Longitudinal study, Heart failure, Stroke, Myocardial infarction

I is well accepted that patients with diabetes mellitus (DM) are at risk for cardiovascular $(\mathrm{CV})$ diseases such as coronary artery disease, left ventricular hypertrophy and dysfunction, heart failure, and stroke. The worldwide prevalence of DM has risen dramatically over the past several decades. Although the need for individualized and timely risk assessment has been emphasized, some DM subjects remain free of cardiovascular (CV) disease while others experience serious CV complications. Several studies have suggested that aggressive pharmacological and nonpharmacological interventions improve CV prognosis in patients with DM. ${ }^{1,2)}$ It may therefore be realistic to select DM subjects at relatively high risk for $\mathrm{CV}$ disease, and then provide intensive risk factor modification to prevent the development of CV events.

Many biomarkers, such as microalbuminuria including urine albumin-to-creatinine ratio (UACR), blood pressure, lipid parameters, and renal function have been put forward as potential indicators of elevated CV risk in individuals with DM. To some extent, however, these variables are of limited use as they are merely mediators for long-term CV injury and are not direct markers of cardiac and vascular abnormality. The natriuretic peptide family, including B-type natriuretic peptide (BNP) and its N-terminal fragment (NT-proBNP), are released from the heart in response to increased intracardiac pressure, cardiac pump dysfunction, hypertensive ventricular hypertrophy, and myocardial ischemia. BNP/NT-proBNP may thus be a direct biomarker for clinical or subclinical cardiac abnormality. In fact, a few community-based studies have shown that increased levels of BNP/NT-proBNP were associated with CV risk and mortality independent of established $\mathrm{CV}$ risk markers. ${ }^{3,4)}$

From the Departments of ${ }^{1}$ Internal Medicine, ${ }^{2}$ Preventive Medicine, and ${ }^{3}$ Neurosurgery, Iwate Medical University, ${ }^{4}$ Department of Health and Physical Education, Iwate University, Iwate, and ${ }^{5}$ The First Anti-tuberculosis Association, ${ }^{6}$ Department of Biostatics, School of Public Health, The University of Tokyo, Tokyo, Japan.

This study was supported in part by grants-in-aid from the scientific research fund of the Ministry of Education, Science, and Culture of Japan (23591059) and the Japan Arteriosclerosis Prevention Fund.

Address for correspondence: Motoyuki Nakamura, MD, Division of Cardiovascular Medicine, Department of Internal Medicine, Iwate Medical University, 19-1 Uchimaru, Morioka, Iwate 020-8505, Japan.

Received for publication February 2, 2012.

Accepted February 27, 2012. 
Several previous studies have shown that, in selected patients with DM treated by diabetologists in teaching hospitals, increased levels of plasma BNP/NT-proBNP were related to risk of all-cause mortality and $\mathrm{CV}$ death. ${ }^{5-9)}$ Although such a simple risk screening approach in the DM population may be more helpful for general practitioners or health care providers as a signal for commencement of evidence-based interventions for DM, the specific value of plasma BNP for predicting increased CV risk in unselected DM subjects in a communitybased population has yet to be established. Moreover, no studies have examined the effects of adding plasma BNP testing to a traditional $\mathrm{CV}$ risk prediction model in these subjects.

The aim of the present study was to determine the level of plasma BNP which marked an increased incidence of CV events in unselected community-based individuals with DM. Further, the effects of adding BNP or UACR to an established $\mathrm{CV}$ risk scoring system were examined in terms of an increase in the area under the curve (AUC) generated from a receiveroperating-characteristic (ROC) analysis.

\section{METHODS}

Study subjects: Participants in the present study were part of the Iwate-KENCO study, and the details of recruitment and measurements in the cohort have been provided in previous reports. ${ }^{10,11)}$ In brief, the original cohort $(n=26,469)$ was recruited from the population living in Ninohe, Kuji, and Miyako districts of northern Iwate prefecture, Japan, during 20022004. Of the original cohort living in Ninohe and Kuji districts ( $n=15,927), 15,394$ subjects $(97 \%)$ underwent BNP measurement (BNP cohort: men 5,288; women 10,106). In the BNP cohort, DM individuals were selected by one or more of the following criteria; 1 ) casual blood glucose level $\geqq 200 \mathrm{mg} / \mathrm{dL}$ or fasting blood glucose $\geqq 126 \mathrm{mg} / \mathrm{dL}, 2$ ) use of antidiabetic agents including insulin, and 3) glycosylated hemoglobin (HbA1c) $\geqq 6.5 \%$ (NGPS). Subjects were excluded from the present analysis for the following reasons: age under 40 or over 90 years; missing data on serum creatinine levels, body mass index (BMI), ECG tracing, and blood pressure data. The final statistical analysis for the DM cohort was therefore performed on 1,059 subjects (men 509; women 550; mean age, 66.1 years). The study protocol was approved by the university ethics committee and by local institutional review committees. All participants gave written informed consent.

Measurements: All subjects used a self-report questionnaire to confirm their medical history, including status (yes/no) of prescribed drugs for hypertension, diabetes, and hyperlipidemia. Smoking status (current, past, or nonsmoker) was also assessed by questionnaire. Systemic blood pressure in a sitting position after at least 5 minutes rest was measured by experienced research staff using an automatic digital device. The measurement was performed twice, with the mean value used for statistical analysis. Casual blood samples were taken from upper arm veins with subjects in a seated position. Samples were collected into vacuum tubes containing ethylenediaminetetraacetic acid (EDTA) or a serum separator gel. Blood glucose was determined using an enzymatic method. Glycosylated hemoglobin (HbA1c) was measured quantitatively using values established by the Japan Diabetes Society (JDS) with an HPLC method (Tosoh, Japan). The value for HbA1c (\%) in this paper was presented as the NGSP equivalent value (\%) calculated by the formula HbA1c $=\mathrm{HbA} 1 \mathrm{c}(\mathrm{JDS})+0.4{ }^{12)} \mathrm{En}-$ zymatic methods were used to measure serum total cholesterol levels and serum creatinine. The eGFR was calculated using the equation $\left[\mathrm{eGFR}\left(\mathrm{mL} / \mathrm{minute} / 1.73 \mathrm{~m}^{2}\right)=194 \times \mathrm{Cr}^{-1.094} \times\right.$ Age $\left.^{-0.287}\right]$ taken from the Modification Diet in Renal Disease Study (MDRD) for the Japanese population. ${ }^{13)}$ Hypertension was defined as systolic blood pressure $\geqq 140 \mathrm{mmHg}$ and/or diastolic blood pressure $\geqq 90 \mathrm{mmHg}$ and/or the use of antihypertensive medication. Hyperlipidemia was defined by a total cholesterol concentration $\geqq 240 \mathrm{mg} / \mathrm{dL}$ and/or use of lipidlowering agents. BMI was calculated as weight $(\mathrm{kg})$ divided by the square of height $(\mathrm{m})$. The established CV risk score was calculated using the Framingham 10-year risk score (FRS) including age, sex-specific cholesterol, HDL cholesterol, systolic blood pressure, diabetes, and cigarette smoking. ${ }^{14)}$

BNP and UACR: For BNP measurement a separate $2 \mathrm{~mL}$ venous blood sample was collected into a test tube containing EDTA acid sodium. Tubes were stored immediately after sampling in an icebox and were transported to the laboratory. They were then centrifuged at $1500 \mathrm{~g}$ for 10 minutes. After separation, the plasma samples were stored frozen at $-20^{\circ} \mathrm{C}$, and BNP levels were measured by direct radioimmunoassay using monoclonal antibodies specific for human BNP (Shionogi, Japan). Cross-reactivity of the antibody was $100 \%$ for human BNP and $0.001 \%$ for human atrial natriuretic peptide. Intra- and interassay coefficients of variation were $5 \%$ and $6 \%$, respectively. The lower detection limit of the assay was $0.05 \mathrm{pg} / \mathrm{mL}$.

A spot urine was also collected, cooled and transported to the laboratory during each afternoon and analyzed on the same day. Urine albumin was assessed quantitatively by an immunonephelometric method ( $\mathrm{N}$-antiserum albumin, Dade Behring) and urine creatinine was measured quantitatively by an enzymatic colorimetric test. Urine albumin concentration (mg/ dL) was divided by urine creatinine concentration and expressed as UACR (mg/g). The accuracy of this ratio to compare with the 24-hour urine sample had been demonstrated in previous papers. ${ }^{15,16)}$ The sensitivity limit for albumin was 6 $\mathrm{mg} / \mathrm{L}$. Interassay and intraassay CV were both within $5 \%$.

Outcome: A follow-up survey assessing the incidence of $\mathrm{CV}$ events was carried out after the baseline study (from 2002 to 2004). We defined CV events as a composite of congestive heart failure, stroke, and myocardial infarction. Hospital admissions for congestive heart failure and myocardial infarction in the cohort were identified by accessing data from the Northern Iwate Heart Disease Registry Consortium. Heart failure was defined by Framingham criteria, ${ }^{17)}$ and registration of myocardial infarction was based on criteria used in the MONICA study. ${ }^{18)}$ Stroke events were identified by accessing the Prefecture Stroke Registration Program conducted by the Iwate Medical Association. Stroke diagnostic criteria in this registry are based on those published by the World Health Organization and defined as the sudden onset of neurological symptoms. ${ }^{19)}$ These registrations were initially performed by attending physicians at each hospital using a registration card after admission. Furthermore, to ensure complete capture of all registrations, researchers visited and checked medical charts and/ or discharge summaries at referral hospitals within the study district. All-cause deaths and migration were confirmed by the official resident registration data issued by the local government offices (October 2009). 
Statistical analysis: Continuous variables are shown as mean $\pm \mathrm{SD}$. To test the relationships between BNP and UACR, logtransformation of each biomarker was performed. DM subjects were divided into quintiles according to their baseline levels of plasma BNP and UACR. To compare results among quintiles, ANOVA or chi-square test was used as appropriate. Follow-up for $\mathrm{CV}$ events was carried out after the baseline study through to March 2009. For analyses of CV incidence, person-years were censored at the date of $\mathrm{CV}$ events, the date of migration from the study area, the date of death, or the end of the followup period, whichever came first. Survival from entry into the study was estimated using the Kaplan-Meier method, followed by a trend test (log rank). Using a Cox proportional hazards regression model, sex-age adjusted hazard ratios (HRs) for these biomarkers with $\mathrm{CV}$ events were assessed. In addition, a multivariable proportional-hazard regression analysis was performed to assess the threshold level for increased risk of $\mathrm{CV}$ events (adjustment factors; sex, age, systolic BP, diastolic BP, $\mathrm{BMI}, \mathrm{HbA1c}, \mathrm{CV}$ history, smoking, hyperlipidemia, atrial fibrillation, estimated GFR, use of antihypertensive drugs, and use of anti-DM drugs). To compare the additive effects of plasma BNP or UACR in conjunction with the FRS in terms of the overall diagnostic accuracy of the tests, ROC curves were constructed and AUC and 95\% confidence intervals (CI) for each ROC curve were calculated. The statistical analysis was performed using SPSS (Version 11.0.1J) or MedCalc (Version 9.6.0.0). A significant difference was defined as $P<0.05$.

\section{RESULTS}

Several clinical characteristics of the DM cohort are shown in Table I. The number of DM cases was 1,059. Mean age was 66 years old, and the mean $\mathrm{HbAlc}$ level was 7.2\%. In this DM cohort, prevalence rates for hypertension, obesity (BMI $\geqq 25.0$ ), current smoking, and atrial fibrillation were $57.2 \%, 9.3 \%, 16.6 \%$, and $3.0 \%$, respectively. The median level of plasma BNP was $16.3 \mathrm{pg} / \mathrm{mL}($ men $=15.3$, women $=18.2$ $\mathrm{pg} / \mathrm{mL} ; P=0.584)$. Among the BNP quintiles, mean age and percentage of using diabetic agents and antihypertensive drugs were higher in the highest quintiles than the lower quintiles. The percentage of subjects using lipid-lowering agents did not differ significantly among the quintiles. Plasma BNP levels were significantly correlated with eGFR $(r=-0.222, P<$

Table I. Baseline Characteristics According to Quintiles of Plasma BNP and UACR in DM Cohort

\begin{tabular}{|c|c|c|c|c|c|c|c|}
\hline & \multirow{2}{*}{$\begin{array}{c}\text { Total } \\
(n=1059)\end{array}$} & \multicolumn{5}{|c|}{ BNP } & \multirow{2}{*}{$P^{*}$} \\
\hline & & $\mathrm{Q} 1(n=211)$ & $\mathrm{Q} 2(n=213)$ & $\mathrm{Q} 3(n=211)$ & $\mathrm{Q} 4(n=212)$ & Q5 $(n=212)$ & \\
\hline Inter quartile level & - & $1.0-4.3$ & $7.2-8.6$ & $14.5-16.3$ & $24.7-28.3$ & $46.5-62.3$ & \\
\hline Age (years) & $66.1 \pm 9.1$ & $60.4 \pm 9.4$ & $63.4 \pm 9.1$ & $66.6 \pm 8.1$ & $68.5 \pm 7.2$ & $71.5 \pm 7.1$ & $<0.001$ \\
\hline Male/Female & $509 / 550$ & $103 / 108$ & $117 / 96$ & $103 / 108$ & $90 / 122$ & $96 / 116$ & 0.114 \\
\hline BMI $\left(\mathrm{kg} / \mathrm{m}^{2}\right)$ & $25.2 \pm 3.7$ & $25.6 \pm 3.8$ & $25.1 \pm 3.3$ & $25.2 \pm 3.7$ & $25.3 \pm 3.8$ & $24.6 \pm 3.7$ & 0.072 \\
\hline Systolic BP (mmHg) & $133 \pm 19$ & $131 \pm 18$ & $130 \pm 20$ & $132 \pm 19$ & $135 \pm 19$ & $137 \pm 20$ & 0.001 \\
\hline Diastolic BP (mmHg) & $76 \pm 10$ & $78 \pm 10$ & $77 \pm 11$ & $76 \pm 10$ & $76 \pm 11$ & $75 \pm 11$ & 0.054 \\
\hline Hypertension (\%) & 57.2 & 45.0 & 50.0 & 56.9 & 64.6 & 69.8 & $<0.001$ \\
\hline drugs $(\%)$ & 39.4 & 25.6 & 33.3 & 41.2 & 44.8 & 51.9 & $<0.001$ \\
\hline Hypercholesterolemia (\%) & 19.2 & 22.8 & 20.7 & 18.5 & 19.3 & 14.6 & 0.294 \\
\hline drugs $(\%)$ & 7.6 & 5.7 & 5.2 & 10.9 & 10.4 & 5.7 & 0.757 \\
\hline Current smoking (\%) & 16.6 & 23.7 & 19.7 & 14.7 & 15.6 & 9.4 & 0.001 \\
\hline Atrial fibrillation (\%) & 3.0 & 1.4 & 0.0 & 0.0 & 2.4 & 11.3 & $<0.001$ \\
\hline Hemoglobin bA1c (\%) & $7.2 \pm 1.4$ & $7.5 \pm 1.6$ & $7.3 \pm 1.5$ & $7.2 \pm 1.2$ & $7.1 \pm 1.3$ & $7.0 \pm 1.1$ & 0.001 \\
\hline Anti-DM drugs (\%) & 45.4 & 35.5 & 40.8 & 49.8 & 47.2 & 53.8 & 0.165 \\
\hline eGFR (mL/minute/1.73m²) & $76.1 \pm 17.5$ & $81.3 \pm 16.7$ & $81.1 \pm 18.9$ & $75.2 \pm 16.6$ & $75.7 \pm 16.2$ & $67.3 \pm 15.3$ & $<0.001$ \\
\hline
\end{tabular}

\begin{tabular}{|c|c|c|c|c|c|c|}
\hline & \multicolumn{5}{|c|}{ UACR } & \multirow{2}{*}{$P^{*}$} \\
\hline & $\mathrm{Q} 1(n=212)$ & $\mathrm{Q} 2(n=212)$ & $\mathrm{Q} 3(n=212)$ & $\mathrm{Q} 4(n=211)$ & $\mathrm{Q} 5(n=212)$ & \\
\hline Inter quartile level & $5.2-8.8$ & $11.5-15.4$ & $19.7-26.3$ & $36.0-60.3$ & $116.2-508.9$ & \\
\hline Age (years) & $63.9 \pm 9.4$ & $64.4 \pm 9.0$ & $66.9 \pm 8.3$ & $68.1 \pm 8.9$ & $67.0 \pm 9.1$ & $<0.001$ \\
\hline Male/Female & $117 / 95$ & $94 / 118$ & $86 / 126$ & $97 / 114$ & $115 / 97$ & 0.008 \\
\hline $\operatorname{BMI}\left(\mathrm{kg} / \mathrm{m}^{2}\right)$ & $24.6 \pm 3.3$ & $24.9 \pm 3.3$ & $24.8 \pm 3.4$ & $25.5 \pm 3.9$ & $25.9 \pm 4.3$ & 0.001 \\
\hline Systolic BP (mmHg) & $125 \pm 17$ & $129 \pm 18$ & $130 \pm 16$ & $138 \pm 19$ & $144 \pm 21$ & $<0.001$ \\
\hline Diastolic BP (mmHg) & $73 \pm 10$ & $75 \pm 10$ & $76 \pm 10$ & $78 \pm 10$ & $81 \pm 11$ & $<0.001$ \\
\hline Hypertension (\%) & 46.0 & 48.6 & 52.8 & 67.8 & 75.9 & $<0.001$ \\
\hline drugs $(\%)$ & 29.7 & 32.5 & 36.3 & 46.9 & 51.4 & $<0.001$ \\
\hline Hypercholesterolemia (\%) & 19.3 & 18.4 & 17.9 & 17.5 & 22.6 & 0.680 \\
\hline drugs $(\%)$ & 9.4 & 5.2 & 9.0 & 7.6 & 6.6 & 0.642 \\
\hline Current smoking (\%) & 18.4 & 15.6 & 15.6 & 16.6 & 17.0 & 0.933 \\
\hline Atrial fibrillation (\%) & 2.3 & 1.9 & 3.3 & 2.8 & 4.7 & 0.493 \\
\hline Hemoglobin bA1c (\%) & $7.0 \pm 1.1$ & $7.1 \pm 1.3$ & $7.0 \pm 1.2$ & $7.2 \pm 1.2$ & $7.8 \pm 1.7$ & $<0.001$ \\
\hline Anti-DM drugs (\%) & 40.6 & 47.6 & 44.8 & 41.2 & 52.8 & 0.224 \\
\hline eGFR (mL/minute/1.73m²) & $75.0 \pm 14.1$ & $76.4 \pm 15.4$ & $76.8 \pm 17.0$ & $77.4 \pm 17.3$ & $75.1 \pm 22.6$ & 0.555 \\
\hline
\end{tabular}

BMI indicates body mass index; BP, blood pressure; DM, diabetes; and eGFR, estimated glomerular filtration rate. * Among quintiles. 
$0.001)$, but not with UACR $(r=0.039, P=0.201)$.

The cohorts were followed for 6,010 person-years. Composite $\mathrm{CV}$ events (heart failure, acute myocardial infarction,
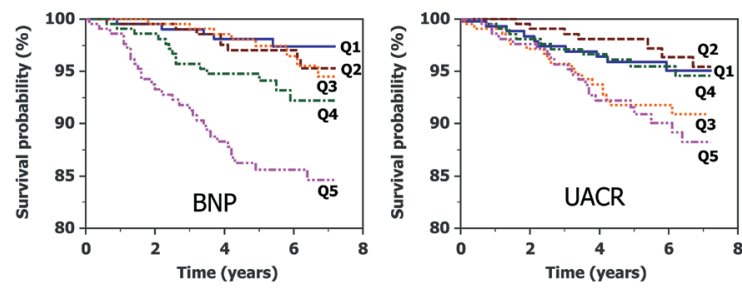

Figure 1. Kaplan-Meier curves of cumulative CV event-free rate according to quintiles of plasma BNP (left) and UACR (right) at baseline in the DM cohort.

Table II. Event Rates and Hazards Ratios for CV Events by Quintiles of Plasma BNP and UACR in DM Cohort

\begin{tabular}{|c|c|c|c|c|c|}
\hline & $\begin{array}{l}\text { CV events } \\
/ 1000 \text { py }\end{array}$ & $\begin{array}{c}\text { Sex-age adjusted } \\
\text { HR }(95 \% \mathrm{CI})\end{array}$ & $P$ & $\begin{array}{l}\text { Multi-adjusted } \\
\text { HR }(95 \% \mathrm{CI})^{*}\end{array}$ & $P$ \\
\hline \multicolumn{6}{|l|}{ BNP } \\
\hline Q1 & 3.9 & Reference & & Reference & \\
\hline Q2 & 6.6 & $1.44(0.47-4.42)$ & 0.552 & $1.68(0.54-5.18)$ & 0.368 \\
\hline Q3 & 6.6 & $1.34(0.43-4.15)$ & 0.612 & $1.51(0.49-4.70)$ & 0.476 \\
\hline Q4 & 11.8 & $2.36(0.83-6.69)$ & 0.106 & $2.26(0.80-6.41)$ & 0.125 \\
\hline Q5 & 27.2 & $\begin{array}{c}4.91(1.82-13.26) \\
P \text { for trend } \\
<0.001\end{array}$ & 0.002 & $\begin{array}{c}4.38(1.69-11.84) \\
P \text { for trend } \\
=0.008\end{array}$ & 0.004 \\
\hline \multicolumn{6}{|l|}{ UACR } \\
\hline Q1 & 7.4 & Reference & & Reference & \\
\hline Q2 & 5.7 & $0.82(0.31-2.21)$ & 0.699 & $0.80(0.30-2.16)$ & 0.657 \\
\hline Q3 & 15.2 & $1.93(0.86-4.32)$ & 0.108 & $1.89(0.84-4.25)$ & 0.124 \\
\hline Q4 & 8.4 & $0.94(0.38-2.33)$ & 0.894 & $0.77(0.30-2.00)$ & 0.600 \\
\hline Q5 & 18.0 & $\begin{array}{c}2.07(0.94-4.54) \\
P \text { for trend } \\
=0.05\end{array}$ & 0.069 & $\begin{array}{c}1.47(0.64-3.40) \\
P \text { for trend } \\
=0.106\end{array}$ & 0.365 \\
\hline
\end{tabular}

*Adjusted by sex, age, systolic BP, diastolic BP, BMI, HbAlc, CV histories, smoking, hyperlipidemia, Af, eGFR, use of antihypertensive drugs, and use of anti-DM drugs. stroke) during the follow-up period (mean $=5.7$ years) were found in 65 cases in the DM cohort. The number of CV events per 1,000 person-years was 10.8. The Kaplan-Meier curves for $\mathrm{CV}$ event-free rate according to quintile levels of BNP and of UACR in the DM cohort are shown in Figure 1. The CV eventfree rate was significantly lower in the highest BNP quintile (IQR; 46.5 to $62.3 \mathrm{pg} / \mathrm{mL}: P<0.001$ by log-rank tests). However, for UACR, although the log-rank test showed a significant difference in $\mathrm{CV}$ event-free curves among the quintiles $(P=0.014)$, no linear relationships were found between the levels of UACR and CV event-free rate (Figure 1). When agestratified analysis (divided by median age) was performed in the case of BNP, similar results were obtained (above median; $P=0.0029$ : below median; $P=0.0021$ ).

As shown in Table II, the number of CV events per 1,000 person-years increased according to quintile levels for both markers. After adjustment for age and sex, Cox regression analysis was performed to analyze the relationship between plasma BNP levels and risk of CV events among the quintiles. The hazard ratio (HR) for the highest quintile of plasma BNP was significantly higher than that for the lowest quintile (HR = 4.91; $95 \% \mathrm{CI}=1.82$ to $13.26 ; P<0.002)$. In addition, in a multivariate adjustment of the model, the relationship between

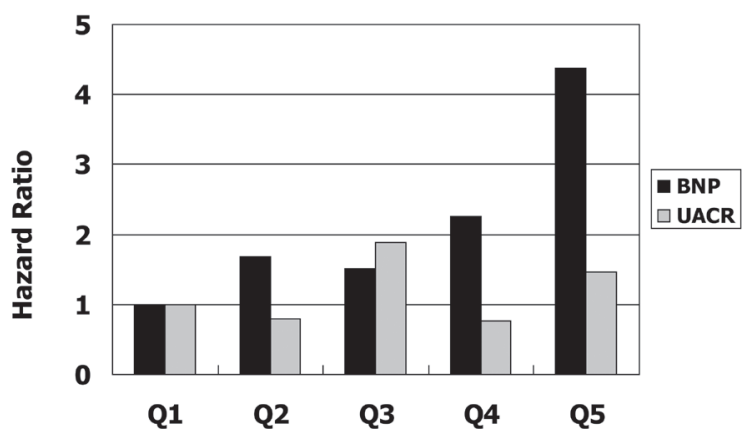

Figure 2. Multivariable adjusted hazard ratios of the risk of $\mathrm{CV}$ events according to quintiles of plasma BNP (black bars) and UACR (gray bars).
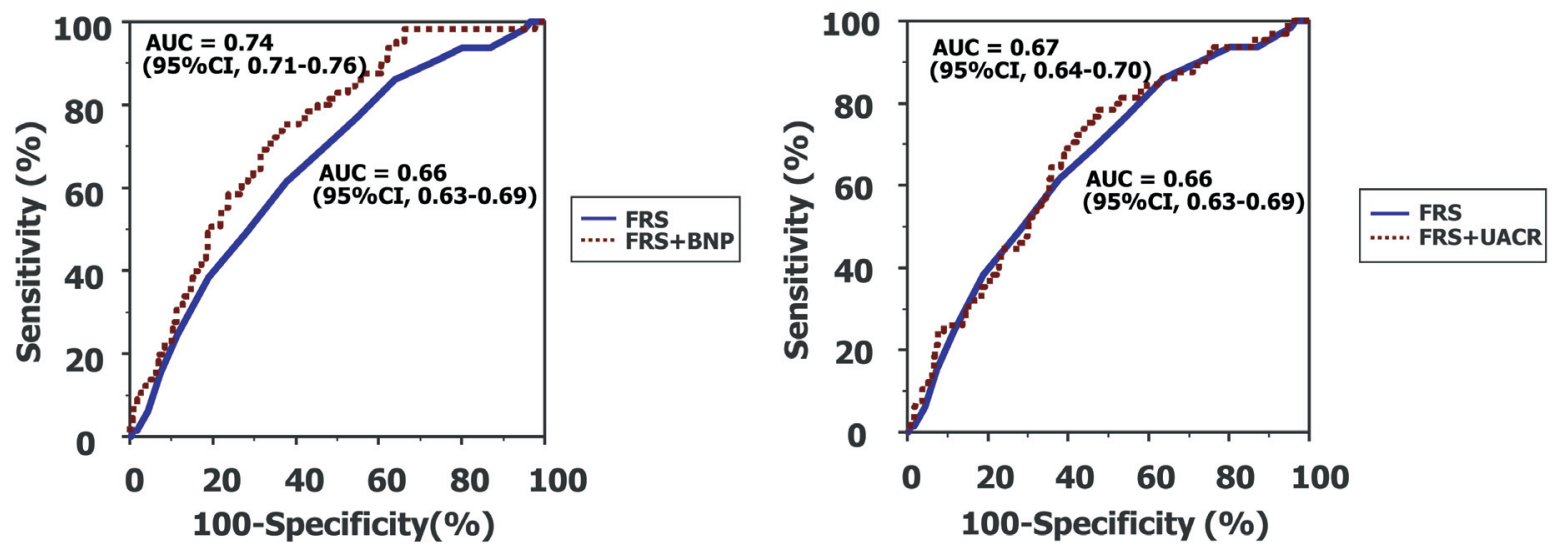

Figure 3. The area under the receiver operating characteristic curves of the Framingham CV risk score with and without BNP (left) and UACR (right) in DM cohort. The area under the curve (AUC) was significantly improved when BNP testing was added to the Framingham CV risk score. 
plasma BNP quintiles and $\mathrm{CV}$ event rate remained robust $(\mathrm{HR}=4.38 ; 95 \% \mathrm{CI}=1.69$ to $11.84 ; P<0.004$ : Table II $)$. As shown in Figure 2, there was a linear association between BNP levels and $\mathrm{HR}$ for $\mathrm{CV}$ events $(P$ for trend $=0.008$ ). However, in the case of UACR, no significant association was found between quintile and $\mathrm{CV}$ events.

The AUC of the ROC analysis for CV prediction was 0.66 (95\%CI, 0.63 to 0.69 ) for FRS and 0.69 (95\% CI, 0.66 to 0.72 ) for BNP (not significant). As shown in Figure 3, when BNP was added to FRS, the ROC analysis AUC $(0.74 ; 95 \% \mathrm{CI}, 0.71$ to 0.76$)$ was significantly increased compared to that for FRS alone $(P=0.05)$. In contrast, when UACR (AUC $=0.61$; $95 \% \mathrm{CI}, 0.58$ to 0.64 ) was added to FRS the overall predictive ability in terms of AUC generated from ROC analysis did not change $(\mathrm{AUC}=0.67 ; 95 \% \mathrm{CI}, 0.66-0.71: P=0.49)$.

\section{Discussion}

The present study has demonstrated that elevated plasma $\mathrm{BNP}$ at 5th quintile levels are useful for $\mathrm{CV}$ risk stratification in the DM population independent of glycemic control and accumulation of established CV risk factors. Moreover, the present findings suggest that the addition of BNP testing to the FRS improves its value for $\mathrm{CV}$ risk prediction.

Tarnow, et al reported that, in 315 patients with type-2 DM, the highest NT-proBNP tertile level had a 2.7 times higher risk for all-cause mortality than the lowest tertile in a longterm follow-up study (19 years). ${ }^{8)}$ Huelsmann, et al have shown that DM subjects with NT-proBNP levels less than 125 $\mathrm{pg} / \mathrm{mL}$ had an excellent short-term prognosis for unplanned hospitalization for CV events and death. ${ }^{5}$ These previous single center studies were performed under specialist diabetes care and did not identify the threshold level of plasma BNP indicating increased risk of $\mathrm{CV}$ events. In a general practical context where health care providers are usually caring for large numbers of patients with DM, it would be helpful to be able to identify individuals at high risk of $\mathrm{CV}$ events by a reliable and simple blood test.

The present study has shown for the first time that a plasma BNP level of approximately $50 \mathrm{pg} / \mathrm{mL}$ is a signal of increased $\mathrm{CV}$ risk in a real world DM population. This value may be a useful marker for the implementation of intensive intervention with multiple drug combinations and behavior modification in these patients. The association between elevated plasma BNP and CV events may be due to the fact that increased plasma BNP is a biomarker for asymptomatic structural heart disease such as impaired left ventricular function, left ventricular hypertrophy, atrial dilatation and fibrillation, valvular heart disease, and myocardial ischemia. ${ }^{20,21)}$ In our previous cross-sectional study applying transthoracic echocardiography in the general population, plasma BNP concentrations over 50 $\mathrm{pg} / \mathrm{mL}$ showed sensitivity and specificity for several select phenotypes of structural heart disease which are prone to progress into several types of $\mathrm{CV}$ events. ${ }^{22)}$

Although obese subjects with insulin resistance as represented by type- $2 \mathrm{DM}$ are expected to have relatively low plasma BNP levels, ${ }^{23)}$ the threshold in this group is higher than in the low risk general population as previously reported. ${ }^{3)}$ The differences in these levels may be due to the relatively high prevalence of left ventricular diastolic dysfunction, hypertro- phy, coronary heart disease, renal dysfunction, and anemia in DM subjects. All of these cardiac and noncardiac factors increase plasma BNP levels, and are obvious substrates to facilitate $\mathrm{CV}$ events.

Urine albumin excretion is suggestive of glomerular permeability, with increasing levels of albumin in the urine indicating extensive glomerular endothelial damage and serving as a marker of systemic microvasculopathy. Several studies have shown that abnormally elevated urinary albumin excretion, microalbuminuria, is predictive of risk for all-cause and $\mathrm{CV}$ mortality in DM subjects. ${ }^{24,25)}$ However, levels of urinary albumin excretion may not be directly related to severity of $\mathrm{CV}$ functional and structural abnormality. In the present results, levels of UACR were not directly correlated with CV event risk in either univariate or multivariate analysis. In contrast, the association between BNP levels and CV events followed a linear dose-effect relationship over time. These findings suggest that plasma BNP levels are a biomarker for a wide range of clinical or subclinical pancardiac target organ damage, ${ }^{26)}$ and thus may be superior to UACR for CV risk stratification in subjects with DM. However, no studies have examined the effect of adding these two biomarkers to an established CV risk prediction model in terms of ability to discriminate between cases and noncases. The present study has demonstrated that the C-statistics of the ROC analysis for CV prediction by BNP testing were comparable to the FRS, and improved significantly when BNP testing was added to the established CV risk model. In view of these findings, plasma BNP measurement appears to be more useful than UACR as a marker of total CV risk in subjects with DM.

The present study had several strengths. The study cohort included a large number of subjects with DM $(>1,000)$. This group may be representative of the real world situation because these subjects were recruited from a community-based population irrespective of anti-DM medication status and glycemic control levels. Plasma BNP measurement was performed in fresh plasma samples without long-term freezing and repeated thawing. CV events were captured prospectively according to previously determined standard epidemiological criteria and confirmed by research staff at medical chart review. Several important biochemical parameters such as UACR and HbAlc at baseline were determined concomitantly before the start of the follow-up study.

Despite the merits delineated above, several limitations must be considered when interpreting the results. First, DM subjects were defined mainly by a single $\mathrm{HbAlc}$ measurement. However, Selvin, et al reported that the within-person coefficient of variation in repeated $\mathrm{HbAlc}$ measurements was less than $4 \%$ in a community-based study. ${ }^{27}$ Second, mean BMI was lower in our study than in previous DM populations. ${ }^{5,8)}$ Cardiac natriuretic peptides in plasma have been reported to be lower in subjects with obesity and insulin resistance than in nonobese and insulin sensitive subjects. ${ }^{23)}$ Consequently, threshold BNP levels may be lower in a predominantly obese DM population. Third, the outcome of this study did not include all types of coronary artery disease such as angina pectoris and sudden cardiac death. Further studies are needed to validate the present results in other ethnic populations with a higher prevalence of coronary artery disease. Lastly, the effects of adding BNP to the FRS in terms of ability to discriminate between cases with and without $\mathrm{CV}$ events were evaluated by 
a less sensitive method such as c-statistics (AUC of ROC analysis) rather than the recently developed event-specific reclassification tables and integrated discrimination improvement. ${ }^{28)}$ However, the present study showed a significant improvement in the AUC. This means that, even using the traditional statistical method, the discriminatory abilities were clearly enhanced by the addition of BNP to the established CV risk model in subjects with DM.

In conclusion, in a community-based sample of people with DM, plasma BNP levels above the 80th percentile are directly associated with $\mathrm{CV}$ risk. Measurement of plasma BNP alone or with an established CV risk score is of value for predicting $\mathrm{CV}$ events in these subjects.

\section{ACKNOWLEDGMENTS}

We thank members of the Northern Iwate Heart Disease Registry Consortium for their help to correct the outcome data: Toshiaki Sakai, MD; Osamu Nishiyama, MD (Ninohe Hospital); Tatsuya Matsumoto, MD (Kuji Hospital); Kenyu Sato, MD; Toshie Segawa, MD; Tomohiro Takahashi, MD (Iwate Medical University). We are grateful to Dr Jo Satoh for his critical reading and comments on this paper.

\section{REFERENCES}

1. Gaede P, Vedel P, Larsen N, Jensen GV, Parving HH, Pedersen O. Multifactorial intervention and cardiovascular disease in patients with type 2 diabetes. N Engl J Med 2003; 348: 383-93.

2. Holman RR, Paul SK, Bethel MA, Matthews DR, Neil HA. 10year follow-up of intensive glucose control in type 2 diabetes. $\mathrm{N}$ Engl J Med 2008; 359: 1577-89.

3. Wang TJ, Larson MG, Levy D, et al. Plasma natriuretic peptide levels and the risk of cardiovascular events and death. N Engl J Med 2004; 350: 655-63.

4. Sakuma M, Nakamura M, Tanaka F, et al. Plasma B-type natriuretic peptide level and cardiovascular events in chronic kidney disease in a community-based population. Circ J 2010; 74: 792-7.

5. Huelsmann M, Neuhold S, Strunk G, et al. NT-proBNP has a high negative predictive value to rule-out short-term cardiovascular events in patients with diabetes mellitus. Eur Heart J 2008; 29 : 2259-64.

6. Bhalla MA, Chiang A, Epshteyn VA, et al. Prognostic role of Btype natriuretic peptide levels in patients with type 2 diabetes mellitus. J Am Coll Cardiol 2004; 44: 1047-52.

7. Gaede P, Hildebrandt P, Hess G, Parving HH, Pedersen O. Plasma $\mathrm{N}$-terminal pro-brain natriuretic peptide as a major risk marker for cardiovascular disease in patients with type 2 diabetes and microalbuminuria. Diabetologia 2005; 48: 156-63.

8. Tarnow L, Gall MA, Hansen BV, Hovind P, Parving HH. Plasma $\mathrm{N}$-terminal pro-B-type natriuretic peptide and mortality in type 2 diabetes. Diabetologia 2006; 49: 2256-62.

9. Tarnow L, Hildebrandt P, Hansen BV, Borch-Johnsen K, Parving $\mathrm{HH}$. Plasma N-terminal pro-brain natriuretic peptide as an independent predictor of mortality in diabetic nephropathy. Diabetologia 2005; 48: 149-55.

10. Takahashi T, Nakamura M, Onoda T, et al. Predictive value of plasma B-type natriuretic peptide for ischemic stroke: a communi- ty-based longitudinal study. Atherosclerosis 2009; 207: 298-303.

11. Ohsawa M, Itai $\mathrm{K}$, Tanno $\mathrm{K}$, et al. Cardiovascular risk factors in the Japanese northeastern rural population. Int J Cardiol 2009; 137: 226-35

12. Seino Y, Nanjo K, Tajima N, et al. Report of the Committee on the classification and diagnostic criteria of diabetes mellitus. J Jpn Diabetes Soc 2010; 53: 450-67.

13. Matsuo S, Imai E, Horio M, et al. Revised equations for estimated GFR from serum creatinine in Japan. Am J Kidney Dis 2009; 53: 982-92.

14. D'Agostino RB Sr, Vasan RS, Pencina MJ, et al. General cardiovascular risk profile for use in primary care: the Framingham Heart Study. Circulation 2008; 117: 743-53.

15. Nathan DM, Rosenbaum C, Protasowicki VD. Single-void urine samples can be used to estimate quantitative microalbuminuria. Diabetes Care 1987; 10: 414-8

16. Schwab SJ, Dunn FL, Feinglos MN. Screening for microalbuminuria. A comparison of single sample methods of collection and techniques of albumin analysis. Diabetes Care 1992; 15: 1581-4.

17. McKee PA, Castelli WP, McNamara PM, Kannel WB. The natural history of congestive heart failure: the Framingham study. N Engl J Med 1971; 285: 1441-6.

18. Tunstall-Pedoe H, Kuulasmaa K, Amouyel P, Arveiler D, Rajakangas AM, Pajak A. Myocardial infarction and coronary deaths in the World Health Organization MONICA Project. Registration procedures, event rates, and case-fatality rates in 38 populations from 21 countries in four continents. Circulation 1994; 90: 583612 .

19. Omama S, Yoshida Y, Ogawa A, Onoda T, Okayama A. Differences in circadian variation of cerebral infarction, intracerebral haemorrhage and subarachnoid haemorrhage by situation at onset. J Neurol Neurosurg Psychiatry 2006; 77: 1345-9.

20. Nakamura M, Hiramori K. Natriuretic peptide measurement as a screening test for overt and subclinical heart failure. Int J Cardiol 2003; 92: 9-15. (Review)

21. Rana BS, Davies JI, Band MM, Pringle SD, Morris A, Struthers AD. B-type natriuretic peptide can detect silent myocardial ischaemia in asymptomatic type 2 diabetes. Heart 2006; 92: 916-20.

22. Nakamura M, Endo H, Nasu M, Arakawa N, Segawa T, Hiramori $\mathrm{K}$. Value of plasma B type natriuretic peptide measurement for heart disease screening in a Japanese population. Heart 2002; 87: 131-5.

23. Khan AM, Cheng $\mathrm{S}$, Magnusson $\mathrm{M}$, et al. Cardiac natriuretic peptides, obesity, and insulin resistance: evidence from two community-based studies. J Clin Endocrinol Metab 2011; 96: 3242-9.

24. Gall MA, Borch-Johnsen K, Hougaard P, Nielsen FS, Parving HH. Albuminuria and poor glycemic control predict mortality in NIDDM. Diabetes 1995; 44: 1303-9.

25. Gerstein HC, Mann JF, Yi Q, et al. Albuminuria and risk of cardiovascular events, death, and heart failure in diabetic and nondiabetic individuals. JAMA 2001 25; 286: 421-6.

26. Struthers A, Lang $\mathrm{C}$. The potential to improve primary prevention in the future by using BNP/N-BNP as an indicator of silent 'pancardiac' target organ damage: BNP/N-BNP could become for the heart what microalbuminuria is for the kidney. Eur Heart J 2007; 28: $1678-82$

27. Selvin E, Crainiceanu CM, Brancati FL, Coresh J. Short-term variability in measures of glycemia and implications for the classification of diabetes. Arch Intern Med 2007; 167: 1545-51.

28. Pencina MJ, D'Agostino RB, Vasan RS. Statistical methods for assessment of added usefulness of new biomarkers. Clin Chem Lab Med 2010; 48: 1703-11. (Review) 